International Journal of Engineering \& Technology, $7(2.19)(2018) 55-60$
International Journal of Engineering \& Technology
SPC
Website: www.sciencepubco.com/index.php/IJET
Research paper

\title{
Improving the Spatial Resolution of Real Time Satellite Image Fusion Using 2D Curvelet Transform
}

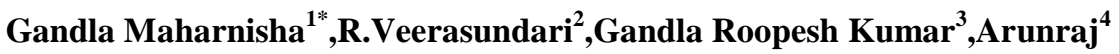 \\ ${ }^{1} U G$ Scholar \\ Department Of Computer Science And Engineering, School Of Computing, \\ Vel Tech Rangarajan Dr. Sagunthala R\&D Institute Of Science And Technology, \\ Avadi, Chennai-600 062, Tamil Nadu, India. \\ ${ }^{2}$ Assistant Professor, Department Of Computer Science And Engineering \\ Meenakshi College Of Engineering \\ Chennai, India \\ ${ }^{3} U G$ Scholar, Department Of Electronics And Communication Engineering \\ Jawaharlal Nehru Technological University \\ Chittoor, India \\ ${ }^{4}$ Assistant Professor \\ Department Of Automobile Engineering, \\ Vel Tech Rangarajan Dr. Sagunthala R\&D Institute Of Science And Technology, \\ Avadi, Chennai-600 062, Tamil Nadu, India. \\ * Maharnisha29@Gmail.Com
}

\begin{abstract}
The fused image will have structural details of the higher spatial resolution panchromatic images as well as rich spectral information from the multispectral images. Before fusion, Mean adjustment algorithm of Adaptive Median Filter (AMF) and Hybrid Enhancer (combination of AMF and ${ }^{\text {Contrast }}$ Limited Adaptive Histogram Equalization (CLAHE)) are used in the pre-processing. Here, conventional Principal Component image fusion method will be compared with newly modified Curvelet transform image fusion method. Principal Component fusion technique will improve the spatial resolution but it may produce spectral degradation in the output image. To overcome the spectral degradation, Curvelet transform fusion methods can be used. Curvelet transform uses curve which represents edges and extraction of the detailed information from the image. Curvelet Transform of individual acquired low-frequency approximate component of PAN image and high-frequency detail components from PAN and MS image is used. Peak Signal to Noise Ratio (PSNR) and Root Mean Square Error (RMSE) are measured to evaluate the image fusion accuracy.
\end{abstract}

Keywords: Fog Computing, IoT, Cloud Computing, Edge Computing

\section{Introduction}

The process of combining relevant information of multiple images of the same object for obtaining a more informative resultant image is called image fusion. The fusion process should not introduce any distortion and the irrelevant features including noise should be eliminated to the maximum level. The term fusion in general means, an approach to extract information that is in several domains. Fusion is used to enhance the satellite image; to visualize the two states in machine vision to analyze the frequency variations of images in robotics; to create a three-dimensional image based on wavelength transform in artificial neural networks and to detect the threads in military areas. Spatial resolution and frequency perspectives related fusion is used to analyze diseases in the medical field [1] [2].

In this paper, Image Fusion has been implemented in Satellite Images. Panchromatic image and multispectral image may not represent more information when the images are individual. Fig. 1 shows the high resolution of the panchromatic image with a low resolution of the multispectral image of Quickbird data of Chennai city. The main aim of satellite image fusion is to increase the spatial resolution while retaining the maximum spectral information of the original multispectral (MS) image. The main objective of this work is

- To combine the high-resolution Panchromatic and lowresolution Multispectral images using fusion which makes use of creating high spatial and high spectral resolution of MS image.

- $\quad$ Before fusion to enhance the images of PAN and MS using Adaptive Median Filter and Hybrid Enhancer which is a combination of AMF and CLAHE 


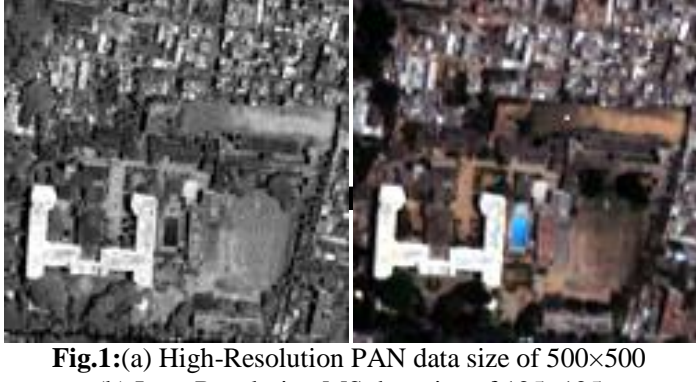

(b) Low-Resolution MS data size of $125 \times 125$

\section{Need for the Study}

Very high-resolution satellite images will have a larger amount of pixels in the image objects which lead more edges. Pixel based fusion (Spatial domain) will introduce more spatial and radiometric (image degradation) errors. Transform domain approaches can handle the spatial distortions very well. Curvelet transform gives better space-frequency characteristics to represent edges. So the Curvelet transform based fusion can reduce radiometric errors i.e. color degradation in the high-resolution images [3].

\section{Literature Review}

Image Fusion techniques combine the spatial details of panchromatic image and color information of Multispectral images to produce a high resolution of Multispectral images. Generation of fused image through manipulation of a set of pixels from the input images is called pixel based fusion which is used to perform segmentation. Feature-based fusion depends on objects and their surrounding salient features like pixel intensities, edges or textures in multiple source images. In decision based fusion, individually processed images from multiple algorithms are merged through decision rules at the high-level concept to obtain fused image [4] [5].Image fusion methods can be categorized according to the data entering the fusion and its purpose. In Multi-view fusion of images, Images are taken from the same modality and taken at the same time but from different viewpoints. In multimodal fusion of images, images are taken by different sensors (e.g. visible and infrared, panchromatic and multispectral satellite images). In a Multitemporal fusion of images, Images are taken at different times in order to detect changes between them. Images of a 3D scene are considered repeatedly with various focal lengths in Multi-focus fusion of images [4].

The image fusion techniques are basically done in two domains like spatial domain Fusion (ex. PCA) and Transform domain Fusion (ex. Curvelet).Pixels values of images are manipulated to get fused image in spatial domain techniques. Images are transformed into frequency domain then fusion operations are performed then the inverse transform is applied to get fused image in frequency domain methods. Spatial domain methods have reduced contrast, spatial distortions, unsolicited artifacts and many drawbacks. Examples of spatial domain methods are Brovey method, Principal Component Analysis (PCA) [6] [7],Intensity-Hue-Saturation (IHS) based methods [1] [8], Restoration-Based Method [9] and high pass filtering based techniques [10].

Like Laplacian transform [11], discrete wavelet transform and Curvelet transform domain approaches [12]can handle the spatial distortions very well. Image fusion based on Wavelet and Curvelet transform method has been proposed in [3]. Curvelet transform has been commonly used in the field of image processing as it gives better space-frequency characteristics and revealed the improvement in PSNR through edge representation [13].
Image Fusion of PAN and MS images using Curvelet transform is implemented with the proposed Hybrid image enhancement in Pre-Processing.

The Performance of modified Curvelet transform based image fusion is compared with conventional PCA image fusion.

Pre-processing is the important step prior to fuse the PAN and MS images. Pre-processing is performed to clear unwanted distortions and to enhance some important image features.Pre-processing steps include Image De-noising, Image Enhancement, Image Registration.

When satellite captures the images, the sensor will introduce noises in the PAN and MS images. This noise can be eliminated by De-noising. Neighboring pixels of one object in PAN and MS images have similar brightness value. Neighboring pixels average value is used to restore distorted pixel in the image. Image Denoising can be performed by Adaptive Median filter algorithm using (1)[14].

$Y(i, j)=\operatorname{median}\{X(i-s, j-t) \mid(s, t) \in W\}$

Let $\mathrm{X}(.,$.$) and \mathrm{Y}(.,$.$) be the input and output respectively, of the$ median filter. Here $\mathrm{W}$ is the window that is defined in terms of image co-ordinates in the neighbourgood of the origin. The total no of points in a window is called window size. $(\mathrm{s}, \mathrm{t})$ are used to find relative row and column positions of moving window.(i,j) are row and column positions of input images.

AMF is used to remove the noise and to reduce distortion. In AMF, noise pixel is identified by comparing and thresholding adjustable neighborhood pixels through spatial processing. Finally, the corrupted pixels are restored using the median value of selected window[15]

Here image enhancement is done using mean adjustment algorithm of Adaptive Median Filter and Hybrid Enhancer which is used to improve the contrast, brightness of images. The steps involved in Mean Adjustment Algorithm of AMF are given below

Step1: Input the Filtered Image

Step2: Case 1: PAN Scale Image

Step3: Set the thresholding values (Median)

Step4: Set lower and upper thresholding values to calculate the Minima and Maxima.

Step5: Apply the double Precision to the Image

Step6: Apply Normalization

Step7: Calculate the Mean of the Gray Scale Value

Step8: Adjust the Mean Value

Step9: Case 2: Multispectral Image

Step10: Set lower and upper thresholding values to calculate the Minima and Maxima

Step11: Color Image Thresholding (Image Bandwidth)

Step12: Convert from Multispectral RGB Image to NTSC Color format (to improve Luminance, the intensity of light emitted from a surface per unit area in a given direction)

\section{Image fusion techniques and design}


Step13: Calculate the Mean adjust value for Green layer using Color Image Upper Thresholding

Step14: Calculate the Mean adjust value for Blue layer using Color Image Lower Thresholding

Step15: For both cases, calculate Minima and Maxima for Layers.

\section{Step16: Compute (Image-Minima/Maxima-Minima)}

Step17: Enhanced Image Output.

The processing Steps for computing CLAHE Algorithm is given below

Some image enhancement operations are performed to enhance the image by each layer. In this contrast enhancement techniques are applied using Contrast Limited Adaptive Histogram Equalization Algorithm. That is extension to the traditional Histogram Equalization technique. The difference between lowest and highest intensity level is called contrast. Increase the contrast value means more difference between lowest and highest intensity level.

Step 1: Contrast enhancement of the images is performed by transforming the values in the intensity image. It operates on tiles (small data regions), rather than the entire image.

Step 2: Contrast Enhancement is performed on each tile, to match the histogram of the output region approximately with specified histogram.

Step 3: To eliminate artificially induced boundaries bilinear interpolation is used to combine the neighboring tiles.

Step 4: To avoid the noise which might be present in the image, the contrast, especially in homogeneous areas can be limited.

Step 5: The traditional Histogram Equalization is defined as the adaptive Histogram Equalization Method.

Step 6: Image contrast to be enhanced by changes the values of the intensity image. Histogram equalization operates on small data regions (tiles), rather than the entire image.

Step 7: Each data region contrast is enhanced, so that the output region of histogram approximately matches the specified histogram.

Step 8: The neighboring tiles are combined by usingtheBilinear interpolation in order to eliminate artificially creating.

Step 9: A good threshold can be selected which leads to an efficient enhancement for fusion.

In the hybrid enhancer, AMF enhanced image is applied to Contrast Limited Adaptive Histogram Equalization (CLAHE). The process involved in hybrid enhancer is given in (2).

$\mathrm{i}_{\mathrm{N}}(\mathrm{x}, \mathrm{y})=\frac{\mathrm{i}_{0}(\mathrm{x}, \mathrm{y})-\mathrm{M}_{1}}{\mathrm{M}_{2}-\mathrm{M}_{1}} \times \mathrm{M}_{\mathrm{g}}$

Where $i_{N}(x, y)$-Hybrid enhancer output image. $i_{0}(\mathrm{x}, \mathrm{y})$-Input image and $M_{1}, M_{2}$-Minimum and Maximum intensity values, $M_{g}$ Histogram applied Image.
Re-sampling or Image Registration is the mathematical technique used to create a new version of the image with a different width and/or height in pixels[16][17]. In re-sampling, increasing the size of an image is called upsampling and reducing its size is called downsampling. Fig. 2 shows the Image registration process for aligning the two or more images of the same object.

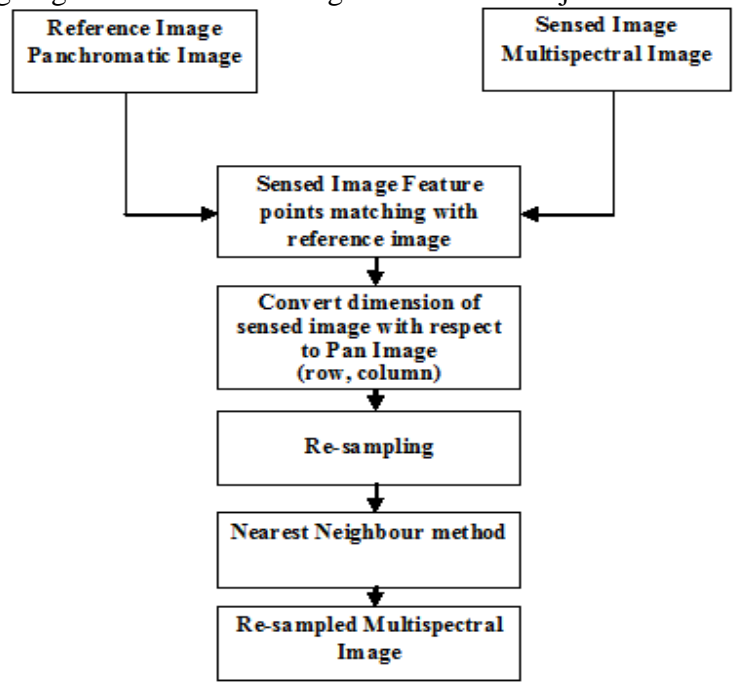

Fig. 2:Flow diagram of image registration.

\subsection{Principal Component Analysis (PCA)}

Multidimensional data sets are reduced to lower dimensions for analysis by PCA. In PCA, eigenvectors are used to transform a number of correlated variables into a number of uncorrelated variables called principal components.PCA may introduce minimal color distortion in Fusion. Fig. 3 shows the Architecture of PCA image Fusion. Implemented PCA procedures are explained below.

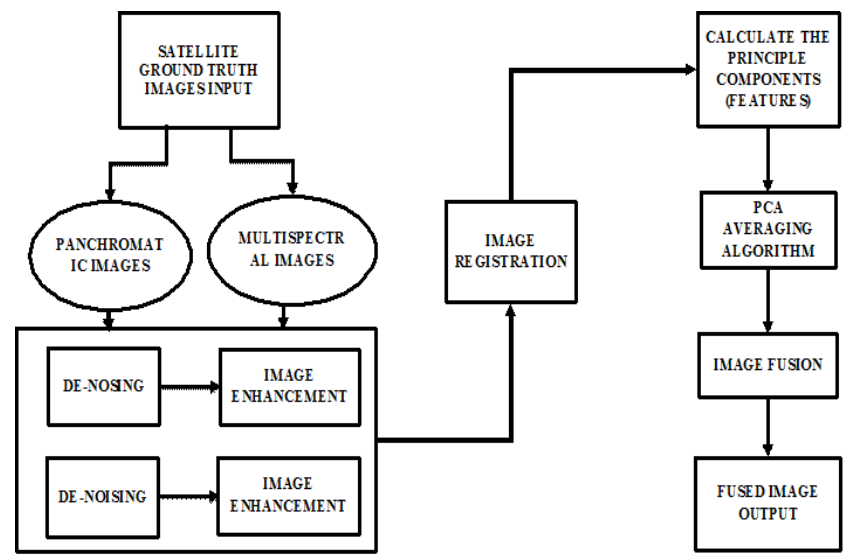

Fig. 3:Architecture of PCA image fusion

Initially, the PAN image and hyperspectral image of R, G and B are read as matrices and converted into column vectors of read images. Then co-variances of column vectors are computed. Covariance matrix will be a $2 \times 2$ matrix. Then Eigen values and Eigen vectors of the covariance matrix are computed and normalized. Based on the descending values of Eigenvalues, the corresponding Eigenvectors are multiplied with image matrix to produce Principle Component. This is the first output of PCA1. The PCA1 values are multiply with first band (Red) of MS image and PAN image. And it is repeated for Green and Blue bands. Then all the bands are concatenated and adaptive histogram equalization is applied to form the fused PCA output.

\subsection{Curvelets}

In Curvelet transform signal can be converted from the time domain into the frequency domain using mathematical operators 
called transformation. To overcome the spectral degradation, Curvelet transform fusion methods can be used.

Curvelet transform uses curve which represents edges and extraction of the detailed information from the image. Fig. 4 shows the architecture of Image Fusion using Curvelet Transform.

Scale, orientation, and translational parameters are used to form Curvelet functions.Scale, orientation, and translational parameters are used to form Curvelet functions. The Curvelet transform has four stages. In Sub Band Decomposition, the Satellite images (PAN and MS) are divided into individual sub-band frequencies (4). This step divides the image into several resolution layers. Each layer contains details of different frequencies. Smooth Partitioning is done by non-negative smooth function windowing $\left(2^{-s} \times\right.$ $2^{-s}$ size of $\left.w\right)$ localized around dyadic squares (5).Renormalization is centering each dyadic square to the unit square $[0$, $1] \times[0,1]$ for each $Q(6)$. Ridgelet is an orthonormal $\operatorname{set}\left\{\rho_{\lambda}\right\}$ for $L^{2}\left(\Re^{2}\right)$ which divides the frequency domain to dyadic nae $|\xi| \epsilon\left[2^{s}, 2^{s+1}\right]$, samples the $\mathbf{s}$ coronae atleast $2^{s}$ times in the angular direction and local wavelets are used for sampling in the radial direction.

$\mathrm{f} \alpha\left(\mathrm{P}_{0} \mathrm{f}, \Delta_{1} \mathrm{f}, \Delta_{2} \mathrm{f}, \ldots\right)$

$\mathrm{Q}_{\left(\mathrm{s}, \mathrm{k}_{1}, \mathrm{k}_{2}\right)}=\left[\frac{\mathrm{k}_{1}}{2^{\mathrm{s}}}, \frac{\mathrm{k}_{1}+1}{2^{\mathrm{s}}}\right] \times\left[\frac{\mathrm{k}_{2}}{2^{\mathrm{s}}}, \frac{\mathrm{k}_{2}+1}{2^{\mathrm{s}}}\right] \in \mathrm{Q}_{\mathrm{s}}$

$\left(\mathrm{T}_{\mathrm{Q}} \mathrm{f}\right)\left(\mathrm{x}_{1}, \mathrm{x}_{2}\right)=2^{\mathrm{s}} \mathrm{f}\left(2^{\mathrm{s}} \mathrm{x}_{1}-\mathrm{k}_{1}, 2^{\mathrm{s}} \mathrm{x}_{2}-\mathrm{k}_{2}\right)$

where $\mathrm{f}$ - image; $\mathrm{P}_{0^{-}}$Low pass filter; $\Delta_{1}, \Delta_{2}$ - Band passes filters $\mathrm{Q}_{\mathrm{s}^{-}}$All the dyadic squares of the grid; $\mathrm{W}$ - smooth windowing function.

\section{Results and Discussions}

The MATLAB scripts are used for processing satellite image These Scripts are tested on different satellite images of QuickBirdSatellite. In this section, the results are presented to demonstrate the performance of enhancements in the low contrast satellite images and fusion of Panchromatic and Multispectral images has been tested on several different satellite images. MSE and PSNR have been used to access enhancement and fusion quality. Mean Square Error (MSE) between the original image and the resultant image is used to determine PSNR.Equations (7) and (8) are used to compute the MSE and PSNR respectively[10].

$M S E=\sum_{j} \sum_{i}[I(i, j)-F(i, j)]^{2} /(m \times n)$

$P S N R=10 \log _{10}([255 \times 255] / M S E) \mathrm{Q}$

Where, I (i, j) represents the original (reference) image and $F(i, j)$ represents the modified image and $i$ and $j$ are the pixel position of the $\mathrm{M} \times \mathrm{N}$ image. MSE is zero when $\mathrm{I}(\mathrm{i}, \mathrm{j})=\mathrm{F}(\mathrm{i}, \mathrm{j})$.

Table 1; show the PSNR and RMSE comparison between without image enhancement and with image enhancement for different raw satellite images using AMF and Hybrid enhancer.

From Table 1, enhancements reduce the RMSE compared with the raw image. So the enhanced images can be applied for the fusion to improve spatial resolution. Hybrid enhancement performs better in this analysis.

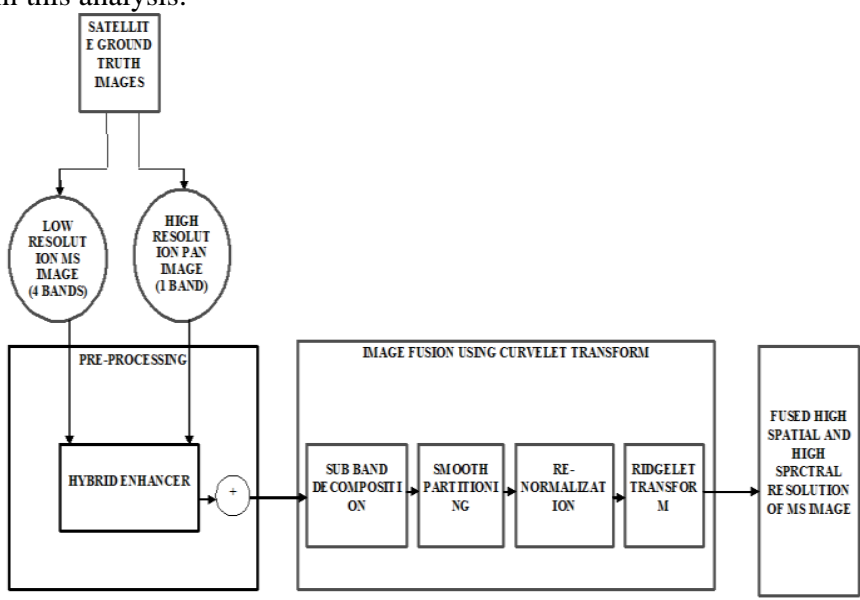

Fig. 4:Image fusion using curvelet transform

Table 1:PSNR and RMSE for various enhancements

\begin{tabular}{|c|c|c|c|c|c|c|}
\hline \multirow[t]{2}{*}{$\begin{array}{l}\text { Image } \\
\text { Name }\end{array}$} & \multicolumn{2}{|c|}{$\begin{array}{l}\text { Without Image } \\
\text { Enhancement }\end{array}$} & \multicolumn{2}{|c|}{$\begin{array}{l}\text { With Image En- } \\
\text { hancement using } \\
\text { Mean Adjust- } \\
\text { ment of AMF }\end{array}$} & \multicolumn{2}{|c|}{$\begin{array}{l}\text { With Image En- } \\
\text { hancement using } \\
\text { Hybrid Enhancer }\end{array}$} \\
\hline & PSNR & RMSE & $\overline{P S N R}$ & RMSE & PSNR & RMSE \\
\hline $\begin{array}{l}\text { Image } \\
\text { 1-MS }\end{array}$ & 24.93 & 0.2091 & 49.67 & 0.0391 & 60.00 & 0.0431 \\
\hline $\begin{array}{l}\text { Image } \\
\text { 1-PAN }\end{array}$ & 23.26 & 0.3072 & 43.34 & 0.081 & 59.00 & 0.0523 \\
\hline $\begin{array}{l}\text { Image } \\
\text { 2-MS }\end{array}$ & 26.31 & 0.1523 & 41.65 & 0.0985 & 60.00 & 0.0429 \\
\hline $\begin{array}{l}\text { Image } \\
\text { 2-PAN }\end{array}$ & 26.57 & 0.1432 & 43.44 & 0.0801 & 58.00 & 0.0506 \\
\hline $\begin{array}{l}\text { Image } \\
\text { 3- MS }\end{array}$ & 24.36 & 0.2383 & 48.58 & 0.0443 & 60.00 & 0.043 \\
\hline $\begin{array}{l}\text { Image } \\
\text { 3-PAN }\end{array}$ & 26.02 & 0.1625 & 40.44 & 0.1125 & 59.00 & 0.052 \\
\hline
\end{tabular}

After PCA and CURVELET image fusions for raw and enhanced images, the results of quality metrics are tabulated in Table 2 and 3. PCA and Curvelet fused images with Hybrid enhancement is given in Fig. 5.

From PCA fusion results of Table 2, Image set 2 has low PSNR value in PCA fusion of unenhanced images. From Table 3 results of Curvelet image fusion, Image set 1 has High PSNR value in Curvelet fusion of hybridly enhanced images.

By analyzing Table 2 and 3, fusions to enhanced images are hav(8) ing better PSNR compared with fusion to raw images.

Table 2:PSNR and RMSE comparison for various PCA imagefusion

\begin{tabular}{|l|l|l|l|l|l|l|}
\hline \multirow{3}{*}{ IMAGE NAME } & \multicolumn{2}{|l|}{\begin{tabular}{l} 
PCA Fusion \\
\cline { 2 - 7 } \\
\end{tabular}} & $\begin{array}{l}\text { Without En- } \\
\text { hancement }\end{array}$ & \multicolumn{2}{|l|}{$\begin{array}{l}\text { With AMF En- } \\
\text { hancement }\end{array}$} & \multicolumn{2}{l|}{$\begin{array}{l}\text { With Hybrid } \\
\text { Enhancer }\end{array}$} \\
\cline { 2 - 8 } & PSNR & RMSE & PSNR & RMSE & PSNR & RMSE \\
\hline $\begin{array}{l}\text { Image 1-Fused } \\
\text { Image }\end{array}$ & 64.91 & 0.0104 & 65.05 & 0.0103 & 66.71 & 0.0096 \\
\hline $\begin{array}{l}\text { Image 2-Fused } \\
\text { Image }\end{array}$ & 57.19 & 0.0162 & 61.14 & 0.0129 & 64.91 & 0.0104 \\
\hline $\begin{array}{l}\text { Image 3-Fused } \\
\text { Image }\end{array}$ & 65.189 & 0.012 & 66.11 & 0.0097 & 67.42 & 0.009 \\
\hline $\begin{array}{l}\text { Average of Fu- } \\
\text { sion Results }\end{array}$ & $\mathbf{6 2 . 4 3}$ & $\mathbf{0 . 0 1 2 9}$ & $\mathbf{6 4 . 1 0}$ & $\mathbf{0 . 0 1 1 0}$ & $\mathbf{6 6 . 3 5}$ & $\mathbf{0 . 0 0 9 7}$ \\
\hline
\end{tabular}

Table 3:PSNR and RMSE comparison for various curvelet image

\begin{tabular}{|l|l|l|l|l|l|l|}
\hline \multirow{2}{*}{ IMAGE NAME } & \multicolumn{9}{l|}{ CURVELET Fusion } \\
\cline { 2 - 7 } & $\begin{array}{l}\text { Without En- } \\
\text { hancement }\end{array}$ & \multicolumn{2}{|l|}{$\begin{array}{l}\text { With AMF En- } \\
\text { hancement }\end{array}$} & \multicolumn{2}{|l|}{$\begin{array}{l}\text { With Hybrid } \\
\text { Enhancer }\end{array}$} \\
\cline { 2 - 8 } & PSNR & RMSE & PSNR & RMSE & PSNR & RMSE \\
\hline $\begin{array}{l}\text { Image 1-Fused } \\
\text { Image }\end{array}$ & 74.01 & 0.0022 & 75.99 & 0.0018 & 76.03 & 0.0017 \\
\hline $\begin{array}{l}\text { Image 2-Fused } \\
\text { Image }\end{array}$ & 73.04 & 0.0024 & 74.72 & 0.002 & 75.67 & 0.0018 \\
\hline $\begin{array}{l}\text { Image 3-Fused } \\
\text { Image }\end{array}$ & 74.13 & 0.0022 & 75.63 & 0.0018 & 75.73 & 0.0012 \\
\hline
\end{tabular}




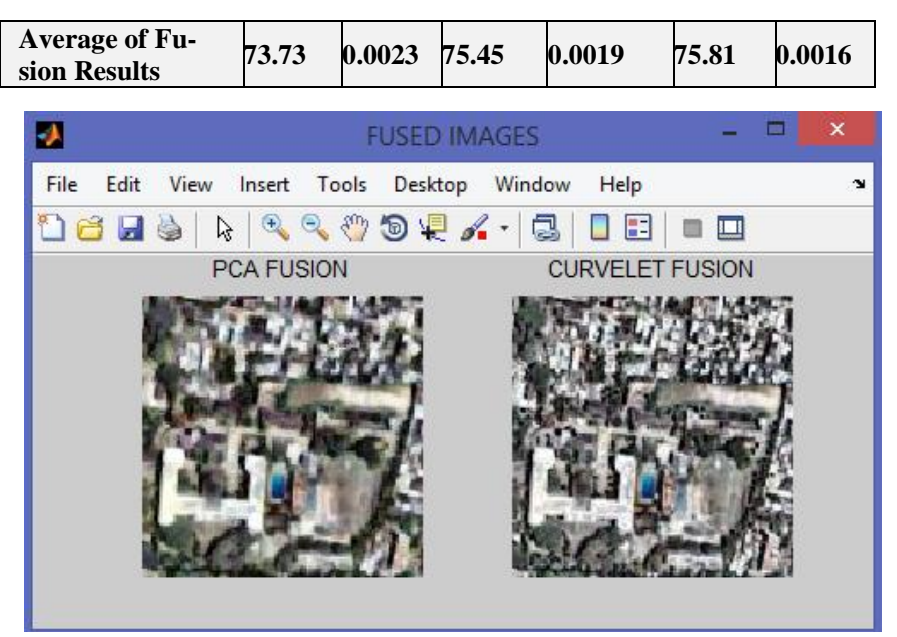

Fig. 5:PCA and Curvelet image fusion

\subsection{Experimental Result Analysis}

Fig.6 and Fig. 7 show the experimental results of various image enhancements and without image enhancement of PSNR and RMSE respectively. Fig. 8 and Fig. 9 show the plots derived from PSNR and RMSE values of various image fusion.

In CLAHE enhancer, adaptive Histogram Equalization is applied for each and every block of the image. In Hybrid enhancer, AMF filter and CLAHE enhancer are combined together for better enhancement of image .PSNR and RMSE are used to analyze the performance of the enhancers.

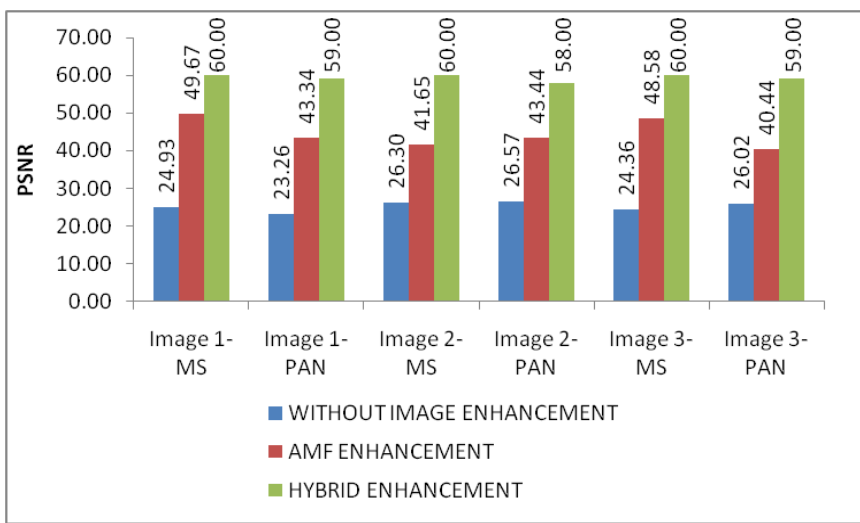

Fig. 6: PSNR values for various image enhancements

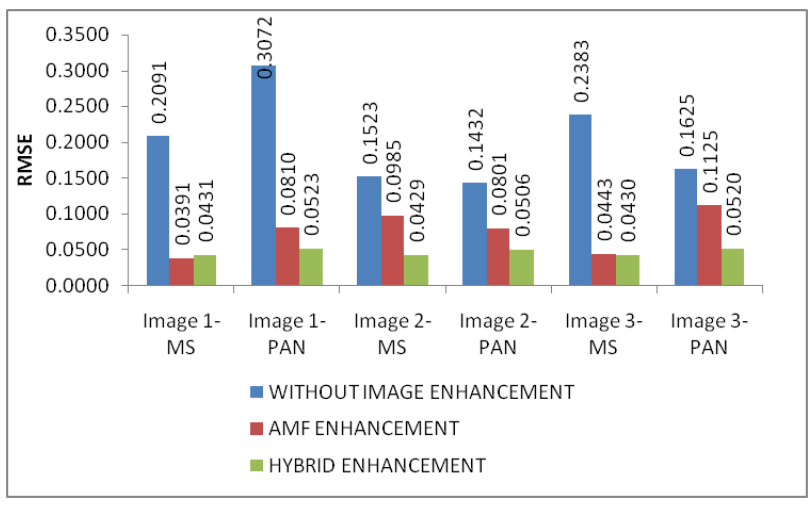

Fig.7: RMSE values for various image enhancements

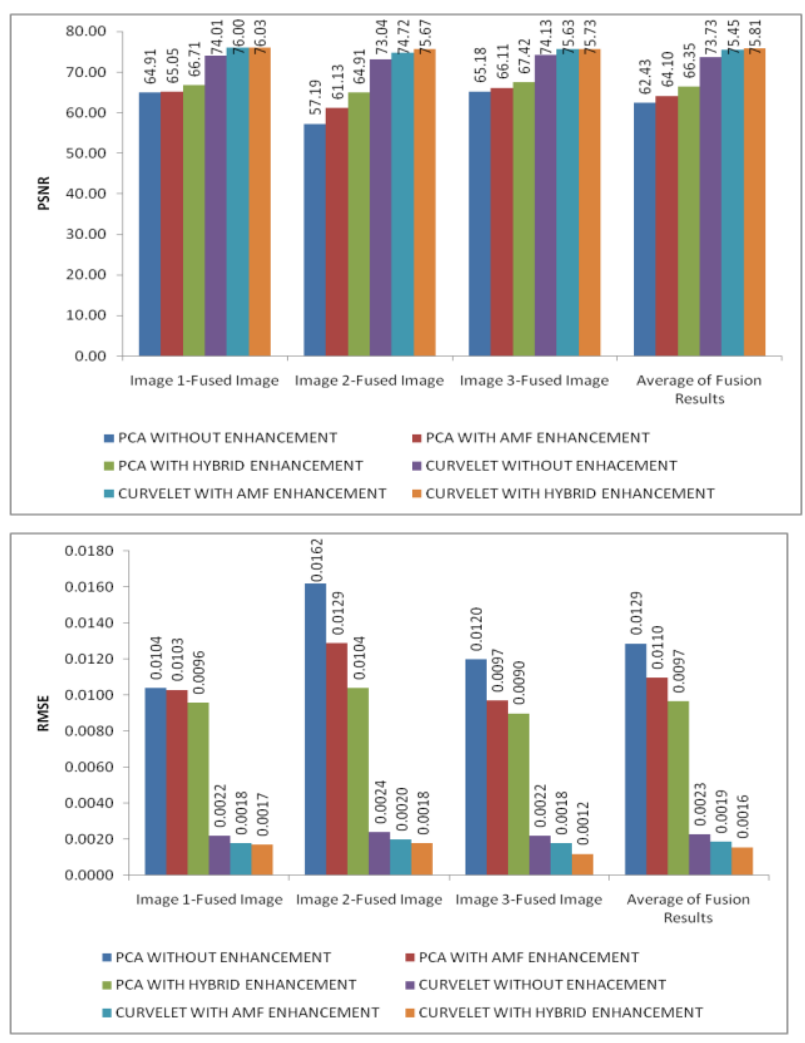

Fig.9 : RMSE values for various image fusions

In the PCA image fusion, the enhanced images are performed better than raw images; especially the hybrid enhanced images have improved the PSNR values. In Curvelet transform fusion, the raw images themselves performed better compared with hybrid enhanced PCA fusion. And hybrid enhances images still improves the fusion rate. So, very high-resolution satellite images like QuickBird can be fused hybrid enhanced images with Curvelet transform.

\section{Conclusion}

The created new image from Pan-sharpening will benefit the highspatial resolution of the panchromatic image and the color information of the Multispectral images. In this research work, PCA and Curvelet based image fusion has been implemented for analysis with AMF and Hybrid Enhancer and these implementations have been tested with very high-resolution Quick-Bird satellite data of Chennai city. From these results, the spatial resolution of the output image is improved with limited distortion.

In future, the image fusion will be implemented for different satellite data with different fusion methods. Further image fusion will be implemented on the real time videos and real time images. It is planned to fuse more than two images and produce a de-blurred fused image and to concentrate on increasing the PSNR values as compared to the current PSNR value.

\section{References}

[1] Harmandeep Kaur, JyotiRani, "Analytical Comparison of Various Image Fusion Techniques,"International Journal of Advanced Research in Computer Science and Software Engineering , vol. 5, issue 5, 2015.

[2] Xiao Xiang Zhu,"A Sparse Image Fusion Algorithm with Application to Pan Sharpening," IEEE Transactions on Geoscience and Remote Sensing, vol.51, no. 5, pp. 2829-2836, 2013. 
[3] Limin Dong, Qingxiang Yang, Haiyong $\mathrm{Wu}$, Huachao Xiao, Mingliang $\mathrm{Xu}$, "High Quality Multi-Spectral and panchromatic image fusion technologies based on Curvelet Transform,"'Elsevier, Wavelet and Curvelet Transform based Image, 2015.

[4] H. B.Mitchell, "Image Fusion-Theories, Techniques and Applications, "Springer-Verlag Berlin Heidelberg, DOI 10.1007/978-3-642-11216-4, 2010.

[5] Umang Thakur, B. Madhushree," A novel approach to Image Fusion using combination of Wavelet Transform and Curvelet Transform,"IJSRD-International Journal for Scientific Research\& Development, Vol. 3, Issue 10, 2015.

[6] V.P.S Naidu and J.R.Raol "Pixel Level Image Fusion using wavelet and Prinicipal Component Analysis," Defence Science Journal, vol. 58, no. 3, pp. 338-352, 2008.

[7] Frosti Palsson, Johannes R. Sveinsson, Magnus Orn Ulfarsson, and Jon Atli Benediktsson, "Model-Based Fusion of Multi- and Hyper spectral Images Using PCA and Wavelets,"IEEE Transactions on Geoscience and Remote Sensing, vol. 53, no.5, 2015.

[8] Myungiin Choi,“A New Intensity-Hue-Saturation Fusion Approach Image Fusion With a Trade off Parameter," IEEE Transactions on Geoscience and Remote Sensing, vol. 44, no. 6, pp. 443-448, 2006.

[9] S. Li and B.Yang, "Fusion of multispectral and panchromatic images using a restoration-based method," IEEE Transactionson Geosciences and Remote Sensing, vol. 47, no. 5, pp. 1482-1491, 2009.

[10] Chen Chen, Yeqing Li, Wei Liu and Junzhou Huang, "Simultaneous Satellite Image Registration and Fusion in a Unified Framework," IEEE TTransactions on Image Processing, vol. 24, no.11, pp. 4213-4224, 2015. 